\title{
Out-of-hospital helmet CPAP in acute respiratory failure reduces mortality: a study led by nurses
}

\author{
G. Garuti1, G. Bandiera², M.S. Cattaruzza33, L. Gelati2, J.F. Osborn³, \\ S. Toscani2, M. Confalonieri4, M. Lusuardi1
}

\begin{abstract}
Out-of-hospital helmet CPAP in acute respiratory failure reduces mortality: a study led by nurses. G. Garuti, G. Bandiera, M.S. Cattaruzza, L. Gelati, J.F. Osborn, S. Toscani, M. Confalonieri, M. Lusuardi.

Background and Aim. Acute respiratory failure (ARF) is a condition that must be treated as quickly as possible. Continuous Positive Airway Pressure (CPAP) is a common method used to treat ARF in hospital.

The main objective of our study was to investigate the effect of CPAP prior to admission to the emergency room, on the reduction of endotracheal intubation, in-hospital mortality and on the length of stay in hospital (HLOS).

Methods. A prospective, observational (non-randomised) study with a historical control group. Data from 3 groups of patients with ARF, irrespective of cause, was collected: pre-hospital CPAP (PHCPAP) group, i.e. 35 patients treated with a helmet CPAP in the ambulance, by trained nurses (mean age, years $80.1 \pm 7.9 \mathrm{SD}$; 14 males); hospital CPAP (HCPAP) group, i.e. 46 patients treated with helmet CPAP in the hospital emergency room (mean
\end{abstract}

age 78.6 $\pm 6.9 \mathrm{SD} ; 27$ males), and a historical control group of 125 patients treated with medical therapy only (mean age 76.7 $\pm 5.5 \mathrm{SD} ; 52$ males). CPAP was delivered via a helmet interface.

Results. Compared with standard medical therapy, helmet CPAP (pre and in-hospital) reduced mortality by $77 \%(p=0.005)$, while pre-hospital helmet CPAP reduced it by $94 \%(p=0.011)$, after adjustment for age, sex, severity of clinical conditions at entry and diagnosis upon admission.

HLOS was reduced, compared with standard medical therapy, by $63.5 \%$ and by $66 \%$ (adjusting for age, sex, severity of clinical conditions at entry and diagnosis at admission) with helmet CPAP (pre and in-hospital) and with helmet CPAP in the ambulance, respectively $(p<0.0001)$.

Conclusions. Treating patients with ARF of any cause, with CPAP by trained nurses, before hospital admission, is safe, reduces mortality and the length of stay needed in hospital.

Monaldi Arch Chest Dis 2010; 73: 4, 145-151.

Keywords: Acute respiratory failure, CPAP, Helmet, Pre-hospital intervention.

1 Pulmonary Rehabilitation Unit, AUSL of Reggio Emilia, S. Sebastiano Hospital, Correggio (RE),

2 Emergency Unit, Hospital of Mirandola (MO),

3 Public Health Dept., "Sapienza” University, Rome,

4 Pulmonary Unit, Azienda Ospedaliero-Universitaria "Ospedali Riuniti" di Trieste, Italy.

Correspondence: Dr. Giancarlo Garuti, Pulmonary Rehabilitation Unit, S. Sebastiano Hospital, AUSL of Reggio Emilia, Via Mandriolo 11, Correggio (RE), Italy; e-mail: garutigi@ausl.re.it

\section{Introduction}

Treatment of acute respiratory failure with non-invasive ventilation support, Continuous Positive Airway Pressure (CPAP) in particular, is increasing in Emergency Medical Services, because it reduces or delays the need for endotracheal intubation [1] but also because of its feasibility by trained nurses early in out-of-hospital settings [2].

CPAP is very practical since it requires limited equipment, which is both small and light and many devices can use pressurised oxygen as the only power source [3].

In most studies in the emergency setting, CPAP was applied to patients with acute cardiogenic pulmonary edema (ACPE) [4]. In ACPE the efficacy of CPAP on ventilation and oxygenation depends on several interdependent mechanisms, such as, an increase in alveolar pressure, a reduction in lung collapsibility, an increase of partial pressure of oxygen, a better $\mathrm{O}_{2}$ diffusion through the alveolar barrier, a squeezing effect on the interstitial lung fluid that is forced toward the vascular and lymphatic beds. Cardiac performance is also improved through a reduction of preload, derived from an increase of intrathoracic pressure, without reducing cardiac output [5].

Kallio et al. retrospectively evaluated CPAP in a mobile intensive care unit on patients with presumed acute severe pulmonary oedema, and found improved oxygenation and lowered respiratory rate, heart rate, and systolic blood pressure [3].

The application of CPAP in COPD and asthma exacerbation is still a matter for debate. A poten- 
tial mechanism for the efficacy of CPAP is that its positive pressure counterbalances the increased intrinsic PEEP (positive end-expiratory pressure) due to the air trapped in peripheral airways. Thus the expiratory time increases allowing for the small airways to empty more easily [6].

CPAP is increasingly applied in critical settings, including out-of-hospital emergency [7] and its relative ease of use means that after adequate training, non medical personnel such as nurses are able to use it.

Articles already published demonstrate the efficacy of the technique, focusing in particular on vital parameters and risk of intubation [1-7]. A recent paper has evaluated its use by non medical personnel, reporting a $30 \%$ reduction in tracheal intubation and $21 \%$ reduction in mortality compared with usual care in selected out-of-hospital patients [2].

\section{Materials and Methods}

\section{Study design}

This is a prospective, observational (non-randomized) study on patients who received CPAP in the ambulance (pre-hospital) or in hospital. A historical control group of patients who received standard medical treatment was also included for comparison.

The aim of this study was to assess whether the early use of CPAP in ambulance, with a standard protocol applied by trained non-medical personnel, can be safe and effective in patients with acute respiratory failure of undefined cause. In particular, we evaluate whether the early use of CPAP in the ambulance by trained nurses can reduce endotracheal intubation, in-hospital mortality and length of stay. Furthermore, the outcomes have been compared to a historical control group who were not treated with CPAP but only with usual medical treatment not defined by a standard protocol. The use of concurrent controls was considered unethical given the evidence for the benefit of CPAP.

This study was carried out at the Emergency Department of the General Hospital of Mirandola (Modena, Italy) which is the referral point of the area and performs about 30,000 interventions per year.

The design of the study was approved by the Institutional Review Board of the hospital.

\section{Patients}

All patients with a diagnosis of acute respiratory failure (independently of cause) in the period $1^{\text {st }}$ January - 30th June, 2006 were eligible for prospective evaluation. They were compared with an historical control group admitted in the period 1st July - 31st December, 2005, before the introduction of CPAP therapy.

Three groups of patients were considered:

1. Pre-hospital CPAP (PHCPAP): patients treated with helmet CPAP in an ambulance by trained nurses.
2. Hospital CPAP (HCPAP): patients treated with helmet CPAP on admission to the Emergency Unit under medical supervision; these subjects arrived at the emergency department spontaneously or with a mobile emergency unit not equipped for CPAP treatment.

3. Historical control group: patients treated with standard medical therapy (drugs and oxygen) at admission to the Emergency Unit under medical supervision.

Patients were enrolled in the study if they fulfilled the following inclusion criteria: paroxysmal dyspnea, respiratory rate $\geq 25$ breaths/minute, $\mathrm{SaO}_{2} \leq 90 \%$ while breathing with $\mathrm{O}_{2}$ bag mask, use of accessory inspiratory muscles, paradoxical breathing.

The clinical and drug history of the patient was registered at home by the nurse.

Patients were excluded from the study if they had at least one of the following: politrauma or facial trauma, history of severe COPD, (i.e. subjects on long-term oxygen or mechanical ventilation or with previous episodes of hospitalisation for acute respiratory failure from an exacerbation), suspected or history of pneumothorax, need for immediate intubation, Glasgow Coma Scale (GCS) $\leq 9$, shock or systolic arterial blood pressure $\leq 90 \mathrm{mmHg}$, inability to protect airways or vomiting, inability to cooperate for psychomotor agitation.

A formal diagnosis was carried out following admission to hospital supported by case history, medical examination and a series of instrumental evaluations. In particular, diagnosis of pneumonia was based on a history of respiratory tract infection and the radiological finding of new pulmonary infiltrate at the chest $\mathrm{x}$-ray. A label of COPD exacerbation required a previous diagnosis of COPD based on spirometric evidence of airways obstruction and exposure to tobacco smoking; a sustained worsening of symptoms (shortness of breath, quantity and color of phlegm) defined an exacerbation. The diagnosis of acute cardiogenic pulmonary edema was made based on symptoms and clinical signs, physical examination, ECG, chest $\mathrm{X}$-ray, echocardiography and laboratory tests including blood gas analysis and specific biomarkers such as pro-brain natriuretic peptide.

\section{Training of personnel}

Nurses involved in the study for CPAP application received at least 2 month training by expert physicians and nurses. This training consisted of 2-hour-sessions, three times per week, including theoretical presentations on respiratory failure and practical training with CPAP devices.

\section{Protocol}

\section{A) CPAP application in ambulance}

At the site of first intervention, paramedical personnel started the procedure according to AMLS (Advanced Medical Life Support) stan- 
dards; arterial blood pressure, electrocardiography, and non-invasive oxygen saturation were monitored. The procedure was explained to patients in order to obtain a verbal consent. An arterial sample for blood gases was taken from the radial artery with the patient breathing air directly in the home or on the ambulance before departure.

CPAP was applied in the ambulance by two trained nurses, before departure. The whole procedure was supervised by telephone, at the emergency centre by a physician, in charge of verifying inclusion and exclusion criteria. The helmet (Castar-Starmed, Mirandola, Italy) was applied to the patient before the ambulance departure, and then connected with a plastic tube to a source which mixes oxygen and compressed air (Flow-meter 546 Starmed, Mirandola, Italy) at flows to be adjusted according to a pre-defined table correlating oxygen and air combined flows with a definite $\mathrm{FiO}_{2}$ (figure 1).

Only one nurse assisted the patients during the transfer to hospital, the second being the designated driver of the ambulance. The nurse regulated the CPAP and the resistance valve to a target $\mathrm{SaO}_{2}$ of at least $92 \%$, progressing according to the following scheme: CPAP $5 \mathrm{cmH}_{2} \mathrm{O}$ and $30 \% \mathrm{FiO}_{2}$, CPAP $7 \mathrm{cmH}_{2} \mathrm{O}$ and $40 \% \mathrm{FiO}_{2}$, CPAP $10 \mathrm{cmH}_{2} \mathrm{O}$ and $50 \% \mathrm{FiO}_{2}$. For safety reasons and technical limitation (maximal oxygen flows obtainable on the ambulance), the upper limit of performance was put at $10 \mathrm{cmH}_{2} \mathrm{O}$ and $50 \% \mathrm{FiO}_{2}$. During the transport to the hospital, the nurse was always in contact with the doctor at the emergency centre, to report vital parameters (arterial blood pressure, $\mathrm{EKG}, \mathrm{SaO}_{2}$ ) and exchange information for medical therapy. An arterial sample for blood gases was taken at 30', 60' and 120' during CPAP treatment. In the geographic area where the study was carried out distances to hospital require in general less than 30 minutes, therefore the arterial blood samples at 60' and 120' were always taken in the Emergency Department.

\section{B) CPAP application in hospital}

The protocol for patient selection and CPAP application was the same as on the ambulance.

The only difference with respect to the previous group was the possibility of increasing the

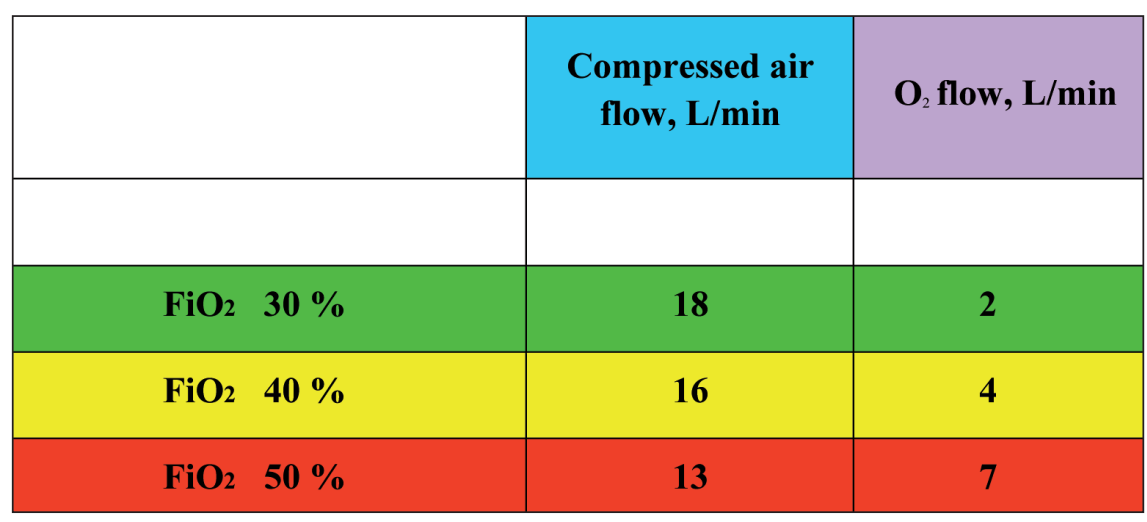

Fig. 1. - Algorithm for the determination of $\mathrm{FiO}_{2}$ with the oxygen / compressed air blender at a final flow of $20 \mathrm{~L} / \mathrm{min}$.
CPAP performance with the same device to 15 $\mathrm{cmH}_{2} \mathrm{O}$ and $60 \% \mathrm{FiO}_{2}$. The monitoring of vital parameters and the timing of arterial blood gases was the same as in the ambulance.

\section{C) Historical control group}

Clinical records of consecutive patients admitted to the emergency department for acute respiratory failure in the 6 months before the CPAP introduction were retrospectively evaluated. At that time, only medical treatment including oxygentherapy, administered with different devices, was available as first line intervention, without any definite protocol.

\section{End-Points}

The main objective of this study was the assessment of the efficacy of Helmet CPAP in the out-hospital setting, defined as improvement of clinical variables, reduction of endotracheal intubation, in-hospital mortality and hospital length of stay. A second objective was to compare the efficacy of CPAP in two different settings: outside the hospital (that is, in the ambulance) and in-hospital, with respect to standard medical therapy. A third objective was to evaluate CPAP as feasible treatment for acute respiratory failure (ARF) from different causes (not only acute pulmonary edema).

\section{Data recording and analysis}

During the study period, data from all patients enrolled was recoded on an Excel worksheet.

In-hospital mortality was cross-checked from clinical records and Hospital Discharge Records.

Descriptive statistical analysis included proportions for qualitative data and means \pm standard deviation (SD) and standard error (SE) for continuous variables; analysis of variance and the chisquared test were used, as appropriate, to evaluate statistical differences among the three groups.

Multiple logistic regression was used to investigate the independent effect of each variable on the outcome (in-hospital mortality) allowing comparisons between groups that can differ significantly at the baseline.

Cox's proportional hazard model was used to evaluate hospital length of stay in relation to the three different treatment groups and to control for potential confounders. All tests and $p$ values are two tailed and statistical significance was set at the $5 \%$ level.

Results
81 patients were treated
with CPAP, 35 in the ambu-
lance (PHCPAP group: 14
males mean age $80.1 \pm 7.9$ SD;
range 54-94 years), and 46


on admission to the emergency room (HCPAP

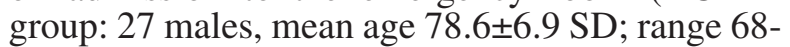
90 years). The historical control group included 125 patients, 53 males, mean age $76.7 \pm 5.5 \mathrm{sd}$; range 58-96 years) (table 1).

The diagnoses at admission are reported for the three groups in figure 2; differences among percentages are statistically significant (Chi squared $=13.4$ with 4 degree of freedom, df; $p=0.001$ ).

Acute cardiogenic pulmonary edema was the most frequent cause of ARF, but varied from $52 \%$ in the control group to $71 \%$ in the PHCPAP. Pneumonia and AECOPD were the second most common diagnoses for HCPAP and Control groups respectively.

Analysis of variance of the three groups in table 1 demonstrated that PHCPAP patients had a baseline heart rate significantly higher, but after 120 minutes the decrease was larger than HCPAP and control patients treated in hospital $(p<0.0001)$.

Baseline values of systolic and diastolic blood pressures did not differ among the 3 groups, but after 120 minutes control patients not receiving CPAP showed significantly higher levels $(p<0.0001$ and 0.002).
The control of the respiratory rate was more rapid in the PHCPAP group, but at 120 minutes there were no significant differences among the three groups.

PHCPAP patients had a more rapid improvement of $\mathrm{SaO}_{2}$ than HCPAP and control patients, particularly important from a clinical point of view at 30 minutes $(p=0.0001)$.

Modifications of $\mathrm{pH}$ were clinically important for the groups treated with CPAP, which at 120 minutes showed a regression of acidosis to $\mathrm{pH}$ values in the normal range, while in the control group acidosis persisted, although with a tendency to improve $(p<0.0001)$.

The three groups showed no significant differences in $\mathrm{PaO}_{2}$ and $\mathrm{PaCO}_{2}$ at baseline. The correction of $\mathrm{PaO}_{2}$ at 120 ' was more evident for the PHCPAP group $(p<0.0001) . \mathrm{PaCO}_{2}$ levels in PHCPAP and HCPAP patients regressed to normal at 120 minutes, while in the control group hypercapnia was still evident, consistent with the acidotic $\mathrm{pH}$.

In table 1 a discrepancy can be noticed between values of $\mathrm{PaO}_{2}$ and oxygen saturation at baseline, since $\mathrm{PaO}_{2}$ is almost the same in the three groups, while $\mathrm{SO}_{2}$ differs by nearly 10 points

Table 1. - Analysis of variance of age and clinical parameters

\begin{tabular}{|c|c|c|c|c|c|c|c|}
\hline & \multirow{2}{*}{\multicolumn{2}{|c|}{$\begin{array}{l}\text { Controls } \\
\mathrm{N}=125\end{array}$}} & \multicolumn{2}{|c|}{ HCPAP } & \multicolumn{2}{|c|}{ PHCPAP } & \multirow{3}{*}{$\mathbf{P}=$} \\
\hline & & & \multicolumn{2}{|c|}{$\mathrm{N}=46$} & \multicolumn{2}{|c|}{$\mathbf{N}=35$} & \\
\hline & mean & SE & mean & SE & mean & SE & \\
\hline Età & 76.74 & 0.57 & 78.65 & 0.95 & 80.06 & 1.08 & 0.0153 \\
\hline HR baseline & 125.39 & 1.61 & 123.46 & 2.66 & 133.80 & 3.05 & 0.025 \\
\hline HR 120' & 85.66 & 1.04 & 95.37 & 1.72 & 88.77 & 1.97 & 0.0000 \\
\hline SBP baseline & 149.84 & 2.27 & 159.67 & 3.75 & 150.57 & 4.29 & 0.077 \\
\hline SBP 120' & 134.28 & 1.01 & 127.83 & 1.67 & 124.11 & 1.91 & 0.0000 \\
\hline DBP baseline & 89.92 & 1.22 & 93.48 & 2.02 & 86.86 & 2.31 & 0.094 \\
\hline DBP 120 & 82.12 & 0.73 & 78.15 & 1.21 & 77.80 & 1.38 & 0.002 \\
\hline RR baseline & 42.50 & 0.56 & 39.72 & 0.93 & 39.97 & 1.06 & 0.013 \\
\hline RR 30' & 36.10 & 0.54 & 34.44 & 0.89 & 32.23 & 1.01 & 0.003 \\
\hline RR 60' & 28.28 & 0.60 & 29.80 & 0.79 & 26.69 & 0.90 & 0.035 \\
\hline RR 120' & 23.39 & 0.99 & 23.04 & 0.75 & 21.89 & 0.86 & 0.456 \\
\hline SO2 baseline & 85.97 & 0.78 & 75.39 & 1.29 & 77.63 & 1.48 & 0.0000 \\
\hline SO2 30' & 89.71 & 0.47 & 88.91 & 0.78 & 93.74 & 0.90 & 0.0001 \\
\hline SO2 60' & 93.12 & 0.34 & 93.24 & 0.56 & 95.97 & 0.65 & 0.0005 \\
\hline SO2 120' & 95.30 & 0.20 & 96.91 & 0.33 & 97.29 & 0.38 & 0.0000 \\
\hline pH baseline & 7.22 & 0.01 & 7.27 & 0.02 & 7.23 & 0.02 & 0.022 \\
\hline pH 30' & 7.24 & 0.01 & 7.33 & 0.01 & 7.29 & 0.02 & 0.0000 \\
\hline pH 60' & 7.28 & 0.01 & 7.35 & 0.01 & 7.33 & 0.01 & 0.0000 \\
\hline pH 120' & 7.29 & 0.01 & 7.37 & 0.01 & 7.36 & 0.01 & 0.0000 \\
\hline PO2 baseline & 49.76 & 0.62 & 47.54 & 1.02 & 48.97 & 1.16 & 0.176 \\
\hline PO2 120' & 99.90 & 4.15 & 104.98 & 6.84 & 138.43 & 7.84 & 0.0001 \\
\hline PCO2 baseline & 45.22 & 1.02 & 48.89 & 1.68 & 46.20 & 1.93 & 0.178 \\
\hline PCO2 120' & 50.10 & 0.88 & 43.00 & 1.44 & 42.60 & 1.66 & 0.0000 \\
\hline SAPS II & 46.13 & 0.49 & 46.37 & 0.81 & 52.20 & 0.93 & 0.0000 \\
\hline
\end{tabular}

$\mathrm{HR}=$ heart rate; $\mathrm{SBP}=$ systolic blood pressure; $\mathrm{DBP}=$ diastolic blood pressure; $\mathrm{RR}=$ respiratory rate. 


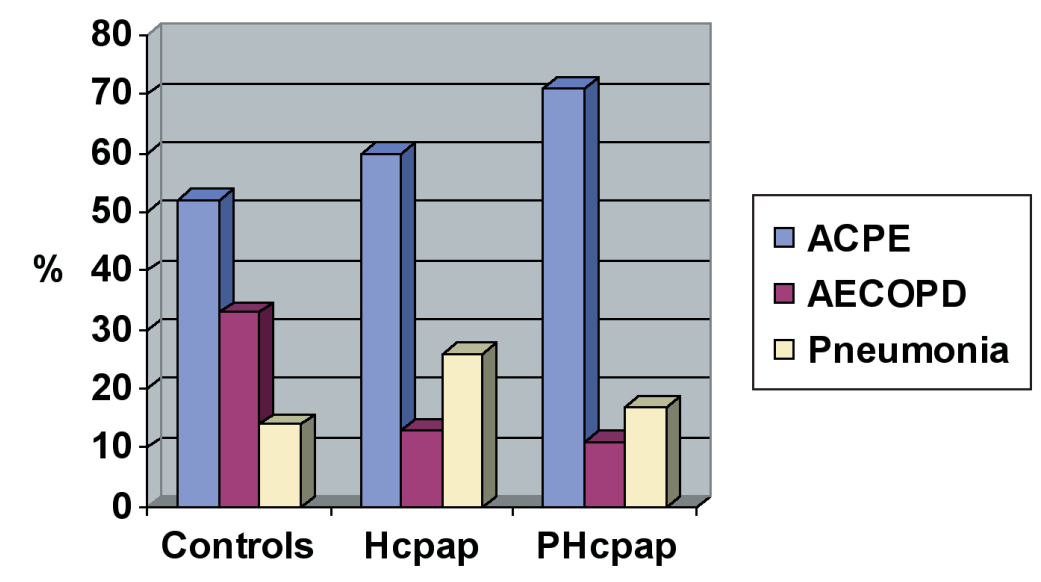

Fig. 2. - Diagnosis at admission for the 3 study groups. (Differences among groups are statistically significant: $C h i$ squared $=13.4$ with $4 \mathrm{df} ; \mathrm{p}=0.001$ )

(85.97 versus 75.39 and $77.63 \%$, respectively). Unfortunately, the retrospective collection of data did not allow us to evaluate in detail those factors either clinical or analytical causing such a discrepancy.

The SAPS II severity score was significantly higher in the PHCPAP group. Baseline SAPS II values did not differ between survivors $(47.3 \pm 5.7$

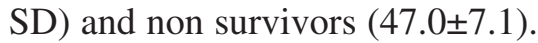

PHCPAP and HCPAP patients maintained a GCS of 15 from baseline to 30 , 60, and $120 \mathrm{~min}-$ utes. On the contrary, patients in the control group showed a modest, but significant deterioration in the same time interval (from 14.8 $\pm 0.08 \mathrm{SE}$ at baseline to $14.3 \pm 0.19 \mathrm{SE}$ at 120 minutes).

No tolerance problems were reported for patients treated with CPAP.

Mortality rates during the hospitalisation period were $24 \%$ for the control group (30 out of 125 patients, despite application of noninvasive ventilation in the hypercapnic patients in the ward), $8.6 \%$ ( 7 out of 81 ) for patients treated with CPAP, with a remarkable difference between the PHCPAP $(2.9 \%$, 1 out of 35) and the HCPAP $(13 \%, 6$ out of 46$)$ patients $(p=0.0097)$.

Logistic regression analysis to evaluate mortality demonstrated that CPAP, independently of the site of application, reduced mortality by $77 \%$ (OR $0.23,95 \% \mathrm{CI}$ $0.08-0.64 p=0.005$ ), as compared to usual drug therapy alone, after adjustment for age, sex, severity of clinical condition at entry (Heart Rate, Respiratory Rate, Glasgow Coma Scale, $\mathrm{pH}, \mathrm{pO}_{2}, \mathrm{pCO}_{2}$ ) and diagnosis at admission.

When we considered the site of application of CPAP, we found that, compared with control, the early application of helmet CPAP in the ambulance, reduced mortality by $94 \%$ (OR $0.06,95 \%$ CI $0.01-0.53, p=0.011)$, after adjustment for sex, age, severity of clinical condition at entry (Heart Rate, Respiratory Rate, Glasgow Coma Scale, $\mathrm{pH}, \mathrm{pO}_{2}, \mathrm{pCO}_{2}$ ) and diagnosis at admission, while the reduction in mortality attributed to the application of CPAP at admission to the emergency department was large, but not statistically significant (OR 0.40, 95\% CI $0.13-1.23, \quad p=0.1097$ ) (table 2).

Diagnosis of pneumonia at admission has 3 times the risk of mortality, independent of sex, age, severity of clinical condition at entry (Heart Rate, Respiratory Rate, Glasgow Coma Scale, $\mathrm{pH}, \mathrm{pO}_{2}, \mathrm{pCO}_{2}$ ) and use of CPAP (OR 3.25, 95\% CI 1.17-9.04, $p=0.024$ (table 2).

We could not evaluate the reduction in intubation rate associated with use of CPAP because in our study no patients needed intubation, either in the HCPAP group or in the PHCPAP group. Intubation occurred only among the patients belonging to the control group (11.2\%), as assessed in the first intervention interval only, from home to the emergency department.

Hospital length of stay (HLOS) was significantly reduced using CPAP compared with pharmacologic treatment alone. The mean length of stay for the control group was 18.8 days ( $\pm 8 \mathrm{SD}$ ) while for HCPAP patients it was 13.9 days $( \pm 9.6$ SD) and for PHCPAP patients it was 12.0 days $( \pm 4.7 \mathrm{SD})(p<0.0001)$.
Table 2. - Logistic regression to evaluate mortality risk in patients treated with CPAP in hospital and in the ambulance (pre-hospital) CPAP versus control group (medical treatment)

\begin{tabular}{lcc}
\hline Independent Variables & OR $(\mathbf{9 5 \%} \mathbf{C I})$ & $\mathbf{P}$ \\
\hline $\mathrm{AGE}$ & $1.04(0.971 .12)$ & 0.2388 \\
$\mathrm{SEX}$ & $0.81(0.341 .90)$ & 0.6244 \\
Baseline Heart Rate & $1.03(1.001 .05)$ & 0.0450 \\
Baseline Respiratory Rate & $1.00(0.941 .08)$ & 0.8927 \\
Baseline Glasgow Coma Scale & $0.76(0.511 .13)$ & 0.1695 \\
Baseline $\mathrm{pH}$ & $8.72(0.09809)$ & 0.3490 \\
Baseline $\mathrm{PaO}_{2}$ & $1.06(0.991 .13)$ & 0.0993 \\
Baseline $\mathrm{PaCO}_{2}$ & $1.01(0.961 .06)$ & 0.6962 \\
AECOPD & $1.36(0.513 .62)$ & 0.5425 \\
Pneumonia & $\mathbf{3 . 2 5}(\mathbf{1 . 1 7} \mathbf{9 . 0 4})$ & $\mathbf{0 . 0 2 4 1}$ \\
Hospital CPAP & $0.40(0.131 .23)$ & 0.1097 \\
Pre-Hospital CPAP & $\mathbf{0 . 0 6}(\mathbf{0 . 0 1} \mathbf{0 . 5 3})$ & $\mathbf{0 . 0 1 1 2}$ \\
\hline
\end{tabular}

$\mathrm{OR}=$ odds ratios $\mathrm{CPAP}=$ Continuous Positive Airways Pressure AECOPD = Acute Exacerbation of Chronic Obstructive Pulmonary Disease. 
Using Cox's proportional hazard model, controlling for sex, age, severity of clinical condition at entry (Heart Rate, Respiratory Rate, Glasgow Coma Scale, $\mathrm{pH}, \mathrm{pO}_{2}, \mathrm{pCO}_{2}$ ) and diagnosis at admission, we found that patients who received CPAP reduced their stay in hospital by $63.5 \%$, $(\mathrm{p}<0.0001)$. In particular, HLOS was reduced by $59 \%$ and by $66 \%$ respectively for HCPAP and PHCPAP groups $(p<0.0001)$. Figure 3 shows the mean values of HLOS.

\section{Discussion}

In the latest 2 decades, treatment of ARF has been modified by the demonstration that non-invasive mechanical ventilation can be as effective as endotracheal intubation, but with reduced probability of adverse events [8,9]. This study is the first comparing the same treatment in two different settings, ambulance and hospital.

This study on efficacy of CPAP confirms that important differences exist in outcomes when using CPAP in comparison with standard medical therapy; indeed in-hospital mortality and hospital length of stay were reduced, showing that CPAP is feasible and has considerable advantages.

In our study, the risk of death was reduced by 94\% if CPAP was applied in the ambulance, while in patients starting CPAP on arrival in hospital, the mortality risk was reduced only by about $60 \%$. This data collates with the most recent randomised controlled study of Thompson [2], that compares pre hospital CPAP with the standard therapy.

Mortality during the full hospitalisation period was $24 \%$ in the control group (despite application of non-invasive ventilation in the post-emergency period for the hypercapnic patients) while for all patients with CPAP, either on the ambulance or in emergency department, it was $8.6 \%$. If CPAP alone were the cause of this difference, then 6-7 patients would need to be treated with CPAP to prevent one death. Both HCPAP and PHCPAP compared to controls showed statistically significant reductions of mortality, indeed, in hospital mortality in the HCPAP group was $13 \%(p=0.03)$ and in the PHCPAP group was $2.9 \%(p=0.005)$;

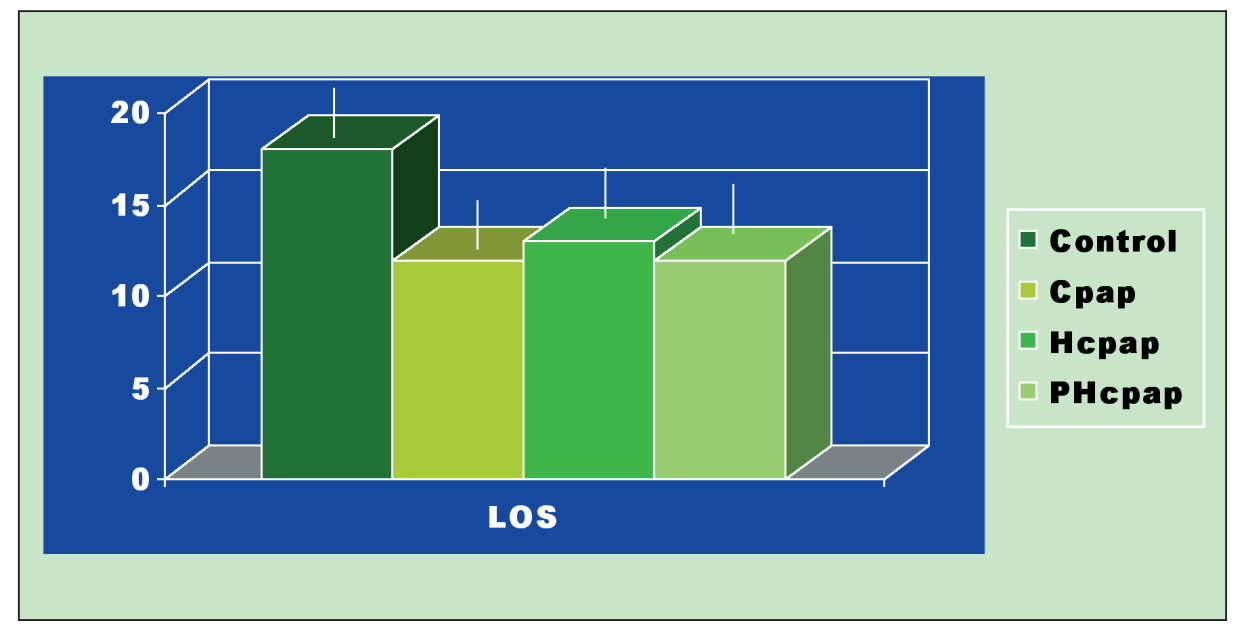

Fig. 3. - Mean Length of Stay (LOS) for controls, Hospital CPAP (Hcpap) patients and Pre Hospital CPAP (PHcpap) patients. Differences between control group and each of the other three groups were significant: $\mathrm{p}<0.0001$. when HCPAP and PHCPAP mortality were compared, the reduction in mortality was not statistically significant, probably due to the small number of deaths observed. Again, if CPAP alone were the cause of these difference, 9 and 5 patients would need to be treated respectively with HCPAP and PHCPAP to prevent one death.

In addition to the potential benefit of CPAP on short-term survival, our data also show a statistically significant reduction of hospital length of stay. There is evidence that early application of CPAP reduces the time spent in hospital by more than $60 \%$. The reduction is even higher $(66 \%)$ for patients receiving CPAP in the ambulance. CPAP reduces hospital stay by 5 and 7 days respectively for HCPAP and PHCPAP, compared with control. Previous studies have shown a trend toward a decrease in HLOS, but were underpowered to detect a statistically significant effect [10].

Major criterions for the success of non-invasive ventilation, and of CPAP in particular, are early intervention [11] and the safe and easy application $[12,13]$; CPAP has been used in different clinical settings [14] including emergency intervention in ambulance, but comparison of outcomes has been made only with controls not using CPAP [10].

This study is the first to compare the efficacy of the early application of CPAP in the ambulance with later application in hospital. It shows that the earlier CPAP is applied, the better the clinical results, the lower the in-hospital mortality and the length of stay. Mortality in the HCPAP group was $13 \%$ while in the PHCPAP group it was $2.9 \%$; if again CPAP alone was the cause of this difference, then 10 patients would need to be treated with CPAP in the ambulance to prevent one death among the HCPAP.

Among the different interfaces that can be used (usually nasal or face masks) the helmet seems particularly suitable for application in the ambulance because it can be easily and rapidly put on, fixing is safe and steady, it requires minimal nursing adjustment, and it is well tolerated by patients (in our study indeed, no patients had problems with the helmet) $[12,13,15]$. The main strength of this study is the evaluation of ambulance CPAP.

Since our study was observational, it had no randomisation and hence, the three study groups statistically differ according to diagnosis at admission and severity of clinical condition at entry (age, heart rate, respiratory rate, $\left.\mathrm{SO}_{2}, \mathrm{pH}, \mathrm{SAPSII}\right)$. Among the three groups, PHCPAP patients were those who had the most disadvantageous conditions at entry, but nevertheless showed the better prognosis. Conversely, it 
must be underlined that the PHCPAP was the group with less pneumonia and AECOPD cases and more ACPE patients, a condition representing the optimal indication for CPAP. Anyway, to study in-hospital mortality we used multiple logistic regression, which analysing the independent effect of each variable on the outcome, allowed comparisons between groups even if they differed significantly at the baseline.

Thus, even if lack of randomisation and comparison with a historical control group means that caution should be used when interpreting the results, it would seem that CPAP, in general has a positive impact on physiologic variables, such as lung gas exchange, acid-base balance, heart rate, respiratory rate, and blood pressure, as has been found in previous studies $[16,17]$.

Several studies have reported on the efficacy of CPAP on acute respiratory failure [18]; a very effective application was on the out-of-hospital treatment of patients with pulmonary oedema [19]. The tertiary objective of this study was to evaluate whether the application of out-of-hospital CPAP is a feasible treatment for ARF of any cause (not only acute pulmonary edema). For this reason, we enrolled patients with ARF of different cause, and in the regression analysis we took diagnosis of admission into account.

Some articles have reported possible negative consequences of a re-breathing effect on $\mathrm{CO}_{2}$ retention, casting doubts about the application of a helmet to patients with hypercapnia or at risk of hypoventilation [20, 21]. Probably, its use for brief periods of time $(<1$ hour) does not produce negative effects on the prognostic course of the illness.

Larger studies are recommended to confirm this apparent benefit of ambulance use of CPAP.

\section{References}

1. Foti G, Sangalli F, Berra L, et al. Is helmet CPAP first line pre-hospital treatment of presumed severe acute pulmonary edema? Intensive Care Med 2009; 35: 656-62.

2. Thompson J, Petrie DA, Ackroyd Stolarz S, Darrel DJ. Out of hospital Continuous Positive Airway pressure ventilation versus usual care in acute respiratory failure: a randomized controlled trial. Ann Emerg Med 2008; 52: 232-241.

3. Kallio T, Kuisma M, Alaspaa A, Rosenberg PH. The use of pre-hospital Continuous Positive Airway Pressure treatment in presumed acute severe pulmonary edema. Prehospital Emergency Care 2003; 7: 209-213.

4. Pang D, Keenan SP, Cook DJ, Sibbald WJ. The effect of positive pressure airway support on mortality and the need for intubation in cardiogenic pulmonary edema. A systematic review. Chest 1998; 114: 1185-1192.

5. Lenique F, Habis M, Lofaso F, Dubois Rande Jl, Harf A, Brochard L. Ventilatory and hemodinamic effects of continous positive airway pressure in left heart failure. Am J Respir Crit Care Med 1997; 155: 500-5.

6. Appendini L, Patessio A, Zanaboni S, et al. Physiologic effects of positive end-expiratory pressure and mask pressure support during exacerbations of chronic obstructive pulmonary disease. Am J Respir Crit Care Med 1994; 149: 1069-76.

7. Sullivan RJ. Prehospital Use of CPAP Emergency Medical Services 2007 (accessed data on EMS responders.com).

8. Hottchkiss JR, Marini JJ. Non invasive Ventilation: an emerging technique for the emergency department. Ann Emerg Med 1998; 32: 470-479.

9. Weitz G, Struck J, Zonak A, Balnus S, Perras B, Dodt C. Prehospital noninvasive pressure support ventilation for acute cardiogenic pulmonary edema. Eur Emerg Med 2007; 14: 276-227.

10. Hubble MW, Richards ME, Jarvis R, Millikan T, Young D. Effectiveness of Prehospital Continuous Positive Airway Pressure in the management of acute pulmonary edema. Prehospital Emergency Care 2006; 10: 430-439.

11. Nava S, Navalesi P, Conti G. Time of non invasive ventilation. Intensive Care Med 2006 32: 361-370.

12. Antonelli M, Conti G, Pelosi P, et al. New treatment of acute hypoxemic respiratory failure: noninvasive pressure support ventilation delivered by helmet - A pilot controlled trial. Crit Care Med 2002; 30: 602-608.

13. Principi T, Pantanetti S, Catani F, et al. Noninvasive continuous positive airway pressure delivered by helmet in hematological malignancy patients with hypoxemic acute respiratory failure. Intensive Care Med 2004; 30: 147-150.

14. Ambrosino N, Vagheggini G. Noninvasive positive pressure ventilation in acute care setting: where are we? Eur Respir J 2008, 31: 874-886.

15. Arnaud Fort P, Boussarie C, Hilbert G., Habachi M. Ventilation non invasive prehopitalier. Presse Med 2002; 31: 1886-1890.

16. McD Taylor D, Bernard SA, Masci K, MacBean CE, Kennedy MP. Prehospital Noninvasive Ventilation: a viable treatment option in the urban setting. Prehospital Emergency Care 2008; 12: 42-45.

17. Patronati N, Saini M, Zanella A, Isgrò S, Pesenti A. Danger of helmet continuous positive airway pressure during failure of fresh gas source supply. Intensive Care Med 2007; 33: 153-157.

18. Nadar S., Prasad N, Taylor RS, Lip GYH. Positive pressure ventilation in the management of acute and chronic cardiac failure: a systematic review and metaanalysis. Internat J Cardiology 2005; 99: 171-185.

19. Kosowsky JM, Stephanides SL, Branson RD, Sayre MR. Prehospital use of continuous positive airway pressure (CPAP) for presumed pulmonary edema: a preliminary case series. Prehospital Emergency Care 2001; 5: 190-196.

20. Patronati N, Foti G, Manfio A, Coppo A, Bellani G, Pesenti A. Head helmet versus face mask for non-invasive continuous positive airway pressure: a physiological study. Intensive Care Med 2003; 29: 1680-1687.

21. Chiumello D, Pelosi P, Carlesso E, et al. Non invasive positive pressure ventilation delivered by helmet vs. standard face mask. Intensive Care Med 2003; 29: 1671-1679. 